\title{
Magnetically controlled superconducting weak links
}

\author{
J. Van de Vondel, ${ }^{1, a)}$ A. V. Silhanek, ${ }^{1}$ B. Raes, ${ }^{1}$ W. Gillijns, ${ }^{1}$ R. B. G. Kramer, ${ }^{1}$ \\ V. V. Moshchalkov, ${ }^{1}$ J. Sautner, ${ }^{2}$ and V. Metlushko ${ }^{2}$ \\ ${ }^{1}$ Institute for Nanoscale Physics and Chemistry (INPAC), Nanoscale Superconductivity and Magnetism \\ Group, K.U. Leuven, Celestijnenlaan 200D, B-3001 Leuven, Belgium \\ ${ }^{2}$ Department of Electrical and Computer Engineering, University of Illinois, Chicago, Illinois 60607, USA
}

(Received 13 May 2009; accepted 24 June 2009; published online 20 July 2009)

\begin{abstract}
We have designed and characterized a magnetic template which can be switched between chains of parallel and antiparallel field distribution by applying an in-plane magnetic field. The parallel field profile creates highly mobile vortex channels in a superconducting film deposited on top, reproducing the behavior of a weak link as evidenced by the presence of Shapiro steps in the current-voltage characteristics under rf excitation. The Josephson coupling can be fully suppressed by changing the field distribution to the antiparallel state. As a result, a reversible ON/OFF switch for magnetically induced weak links has been demonstrated. (C) 2009 American Institute of Physics. [DOI: $10.1063 / 1.3180492$ ]
\end{abstract}

Josephson junctions consist of a weak link between two superconducting reservoirs of paired electrons. Commonly, these junctions are predefined static tunnel barriers that can no longer be modified after fabrication. In the late 1970s Dolan and Lukens ${ }^{1}$ demonstrated that more flexible weak links could be achieved by depleting the superconducting order parameter locally by a highly inhomogeneous magnetic field. This concept of junction has been later on further explored by Clinton and Johnson ${ }^{2,3}$ using the intense stray field generated by a ferromagnetic bar. In all these systems a nodal line of zero order parameter is created by a local field exceeding the upper critical field $H_{c 2}$ of the superconducting material.

In contrast to these static junctions, localized fieldinduced quasiparticle creation can also be achieved by moving vortices. Indeed, a fast moving vortex creates an excess of quasiparticles behind its core thus generating a wake of depleted order parameter that attracts other vortices. ${ }^{4,5}$ These vortices, known as kinematic vortices, have a highly anisotropic vortex core along the direction of motion. Above certain critical velocity, this vortex chain evolves into a phase slip line..$^{5-7}$ As has been shown by Sivakov et al., ${ }^{7}$ these phase slip lines give rise to clear Josephson junction behavior.

The most distinctive feature characterizing a weak link is the presence of a step pattern in the $I(V)$ curves under microwave irradiation. These so-called Shapiro steps result from the ac Josephson relation between the oscillations of the superconducting phase and the associated voltage difference. Similar coherent effects can be obtained by a moving Abrikosov vortex lattice which also gives rise to phase oscillations between two fixed points as a result of the vortex flow. ${ }^{8}$ As early pointed out by Likharev, ${ }^{9}$ all these effects have a common nature that makes it possible to generalize the original definition of the Josephson effect.

In this work we combine previous approaches by creating parallel lines of inhomogeneous field, perpendicular to the superconducting film, which facilitates the hopping of vortices and give rise to clear Shapiro steps in the current-

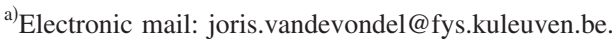

voltage characteristics under rf excitation. This dynamically induced weak link can be switched from ON to OFF when each of the intense magnetic field chains is changed from a parallel to an antiparallel field distribution. Interestingly, the dynamic response of the superconducting system is dominated by the internal vortices generated by the micromagnets rather than from the vortices induced by the external field.

The sample investigated consists of a $40 \mathrm{~nm}$ thick $\mathrm{Al}$ film insulated by a $\mathrm{Si}(3 \mathrm{~nm})$ layer from a periodic pattern of orthogonal cobalt bars, as shown in Fig. 1(a). A reference coevaporated Al plain film has a critical temperature at zero field of $T_{c 0}=1.269 \mathrm{~K}$ and a coherence length $\xi(0)$ $\sim 161 \mathrm{~nm}$. The Co bars have a thickness of $20 \mathrm{~nm}$ and lateral dimensions of $0.3 \times 3 \mu \mathrm{m}^{2}$. The gap between two consecutive bars is $300 \mathrm{~nm}$ [see Fig. 1(a)]. The particular combination of aspect ratio and size ensure a single domain state ${ }^{10}$ in each bar. Figures 1(a) and 1(b) show a schematic representation of the magnetic moment distribution for two distinct remanent states, antiparallel $(a)$ and parallel $(p)$ state, respectively. It is possible to switch between these two magnetic states by applying an in-plane field parallel or perpendicular to the lines formed by the adjacent magnetic poles. The ferromagnetic template has an in-plane coercive field of $50 \mathrm{mT}$ and the saturation magnetization is reached at 100 $\mathrm{mT}$. Simulations of the equilibrium magnetic state performed within the object oriented micromagnetic framework (OOMMF) are shown in Figs. 1(c) and 1(d) for the $a$ and $p$ state, respectively. ${ }^{11}$ Only the out-of-plane $(z)$ component is plotted in both figures since in the thin film limit it has a much stronger influence on the superconductor compared to the in-plane component. ${ }^{12}$ In addition, it is shown that the $z$ component of the field piercing the superconductor reaches a maximum intensity of about $125 \mathrm{mT}$ at the extreme of the bars. This field amply exceeds the upper critical field, $H_{c 2}(0) \sim 20 \mathrm{mT}$ of the Al film deposited on top of the bars thus fully suppressing the superconducting order parameter nearby the magnetic poles. We have corroborated this prediction by measuring a single $\mathrm{L}$-shape $\mathrm{Co} / \mathrm{Si} / \mathrm{Al}$ trilayer of dimensions similar to an individual building block of the array. In this case, no superconducting transition is observed down to the lowest explored temperature of $0.3 \mathrm{~K}$. In a thin super- 

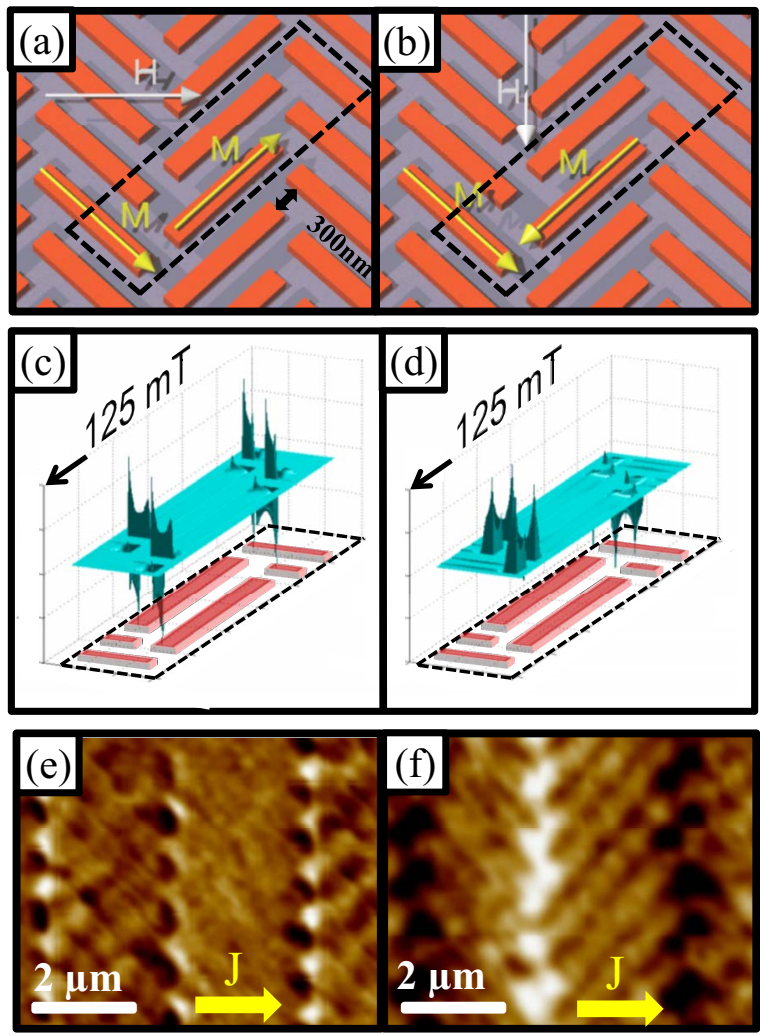

FIG. 1. (Color online) The upper panels show a schematical representation of the magnetization procedure to obtain an antiparallel (a) and parallel (b) field distribution in an array of in-plane magnetic dots. The dashed line indicates the area plotted in panels (c) and (d). The middle panels show OOMMF simulations of the out-of-plane component of the stray field generated by the magnetic template at remenance. Panels (c) and (d) correspond to the moment distribution shown in panels (a) and (b), respectively. Panels (e) and (f) show magnetic force microscopy images of the induced field distributions for an area and magnetic moment distribution coinciding with panel (a) and (b), respectively. White and black indicates magnetic fields with opposite polarities. The arrows define the direction of the injected current applied in the transport measurements.

conducting film these strong out-of-plane stray fields will, at least, generate one internal vortex (antivortex) at each positive (negative) magnetic pole.

Direct visualization at zero applied field, using magnetic force microscopy, of the stray field generated by the magnetic template is shown in Fig. 1(e) for the $a$ state and in Fig. $1(\mathrm{f})$ for the $p$ state. The dark (light) areas represent perpendicular field components that are oriented down (up), with black (white) representing the largest magnitudes. These images directly show that the labels antiparallel $(a)$ and parallel $(p)$ refer to the relative orientations of the out-of-plane components of the fringe fields along the lines formed by adjacent magnetic poles. The field profile along the onedimensional lines of maximum field shows that both magnetic states, $a$ and $p$, exhibit similar intensity of the stray field, as anticipated by the micromagnetic simulations. The minimum distance between neighboring channels is about $2.1 \mu \mathrm{m}$. The out-of-plane stray fields will have a strong influence on the superconducting properties of the thin film, especially the dynamics of internally and/or externally induced vortices. As shown from the transport measurements this leads to a strong dependence of dissipation on the relative orientation of the out-of-plane components.

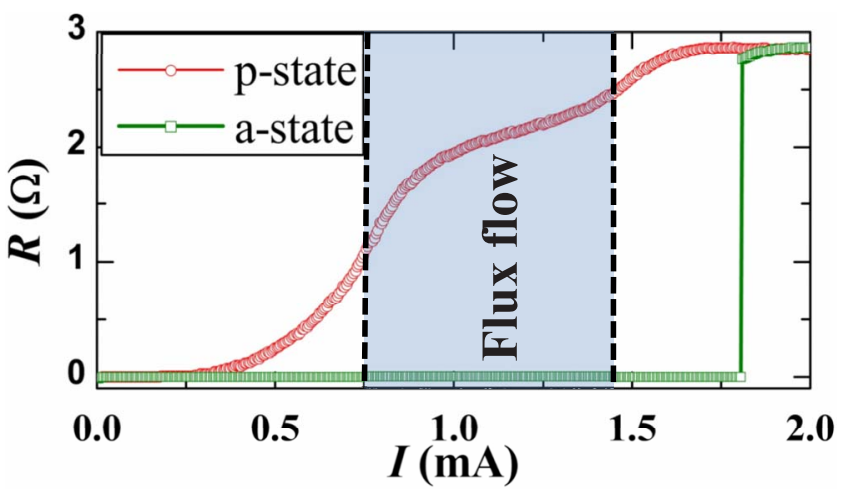

FIG. 2. (Color online) Resistance $R$ vs current $I$ curves for the $a$ and $p$ state at $T=0.7 \mathrm{~K}$ and $H=-0.5 \mathrm{mT}$.

In order to demonstrate the feasibility of the proposed design, we measured current-voltage $V(I)$ characteristics for both magnetic states, with a current applied perpendicular to the channels [see arrows in Figs. 1(e) and 1(f)]. A positive (negative) magnetic field, applied perpendicular to the superconducting thin film, externally generates vortices (antivortices) inside the superconductor. As a result, the total amount of vortices, which is a combination of the internally induced vortices/antivortices and the externally generated vortices/ antivortices, can be tuned at will. The magnitude of the applied field is small $(<5 \mathrm{mT})$ and therefore it will not alter the magnetic state of the ferromagnetic structure. Figure 2 shows a typical set of resistance versus current, $R(I)$, curves at an external field $H=-0.5 \mathrm{mT}$ and temperature $T=0.7 \mathrm{~K}$. Other fields and temperatures show similar features. For the $a$ state, the superconducting film goes abruptly from a nondissipative state at low currents to the normal state at $I$ $\sim 1.77 \mathrm{~mA}$. A completely different behavior is seen when the magnetic template is switched to the $p$ state. In this case, between the nondissipative regime and the normal regime a plateau of intermediate dissipation values indicates the presence of a flux-flow regime of highly mobile vortices. The smooth transition from the pinned vortex lattice (nondissipative regime) to the flux flow regime coincides roughly with $50 \%$ of the normal state resistance. The destruction of the flux flow regime is marked by a $90 \%$ criterion of the normal state resistance. The difference between $p$ and $a$ state indicates that the dissipative state is highly sensitive to the relative orientation of the neighboring magnetic poles. ${ }^{13}$

Figure 3(a) summarizes the field dependence of the different dynamical regimes, using the same criterion as Fig. 2, obtained for the magnetic structure in the $p$ state. Additionally, the onset of vortex motion is marked by a $10 \%$ criterion of the normal state resistance. Note that the existence of a flux flow regime even at zero applied field is a clear indication of the presence of internally induced vortex channels in the $p$ state. ${ }^{3,14}$

There are two characteristic behaviors resulting from the phase coherence across a weak link, the Fraunhofer-like pattern of the critical current as a function of field, and the presence of Shapiro steps in the $V(I)$ characteristics. The fact that the dynamics of our system is dominated by the strongly inhomogeneous stray field generated by the magnetic template suggests that the Fraunhofer-like pattern in $I_{c}(H)$ can be severely modified. ${ }^{15}$ However, the presence of voltage steps in the $V(I)$ curves should still be present. 

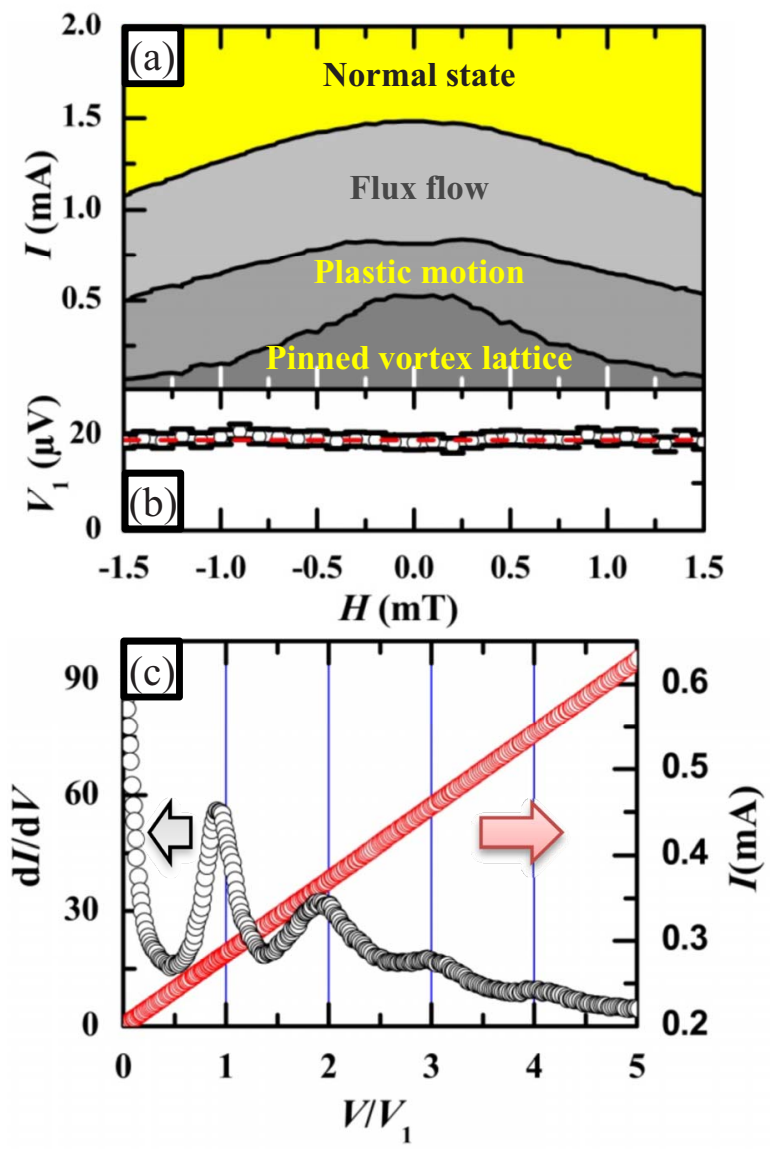

FIG. 3. (Color online) Dynamical properties of the vortex lattice in the $p$ state. (a) Determination of the dynamical phases of the vortex lattice at 0.7 $\mathrm{K}$ with a current of $100 \mu \mathrm{A}$ applied. (b) Magnetic field dependence of the first Shapiro step $V_{1}$. (c) $I$ and $d I / d V$ as a function of the applied voltage at $T=0.75 \mathrm{~K}, H=1 \mathrm{mT}$ and an ac modulation of $100 \mathrm{MHz}$.

In order to check this prediction, we repeated the $V(I)$ measurements with a superimposed high frequency signal of $100 \mathrm{MHz}$. Figure 3(c) shows the current $I$ together with the differential conductivity $d I / d V$ as a function of the applied voltage $V$ at $T=0.750 \mathrm{~K}$ and $H=1 \mathrm{mT}$. The $I(V)$ curve shows a linear dependence with a very weak oscillation amplitude which becomes more evident in the $d I / d V$ response, measured independently with a lock-in technique. These Shapiro steps have a voltage separation of $V_{1}=19 \mu \mathrm{V}$, which indicates commensurability between the external applied frequency and the internally generated frequency, coming from the motion of vortices in the periodic landscape. The fundamental step in the $d I / d V$ curve corresponds to a single step of the vortex lattice in each contributing row or, in other words to a $2 \pi$ change in the phase difference across the channels. Due to the Josephson relation the change in phase difference gives rise to a voltage $V_{\text {single }}=0.207 \mu \mathrm{V}$ across each channel. Since the magnetic channels are perpendicular to the bridge the total voltage drop is given by $V_{1}$ $=m V_{\text {single }}$, where $m$ is the number of contributing magnetic stripes. From this equation, we estimate $m=92$. Since the total amount of channels (irrespective of their field polarity) is $m_{\text {total }}=170$, we have concluded that approximately half of them are contributing to the lock-in mode.

The most plausible explanation for this effect is the deactivation or immobilization of one type of rows by the external field. The rows of antivortices (vortices) emanating from the magnetic bars are partially compensated by the incoming vortices (antivortices), created by a positive (negative) applied field. This compensation produces vacancies in one of the rows thus destroying its high mobility. Since under these circumstances the external field does not contribute to the detected mode locking, the field steps $V_{1}$ should be field independent, in agreement with the $V_{1}(H)$ dependence shown in Fig. 3(b).

Summarizing, we have introduced a magnetic template with well defined chains of strong stray field, which can be readily manipulated to arrange them in either a parallel or antiparallel field distribution. The parallel field profile creates highly mobile vortex channels, which result in coherent phase oscillations across them. This behavior resembles the dynamics of superconducting weak links created by field induced quasiparticles. We have shown that the antiparallel field profile drastically increases the pinning efficiency of the superconductor/ferromagnet structure and destroys the easy flow of vortices. To unveil the ultimate mechanism of vortex hopping and vortex-antivortex annihilation effects theoretical simulations, within time dependent Ginzburg-Landau formalism, would be necessary.

The authors are thankful to V. Gladilin for useful discussions. This work was supported by the Methusalem Funding by the Flemish Government, NES-ESF program, the Belgian IAP, the Fund for Scientific Research-Flanders (F.W.O.Vlaanderen). V.M. acknowledges funding support from U.S. NSF Grant ECCS-0823813. A.V.S., W.G., and J.V.d.V. are grateful for the support from the FWO-Vlaanderen.

${ }^{1}$ G. J. Dolan and J. E. Lukens, IEEE Trans. Magn. 13, 581 (1977).

${ }^{2}$ B. Clinton and M. Johnson, J. Appl. Phys. 85, 1637 (1999).

${ }^{3}$ B. Clinton and M. Johnson, Appl. Phys. Lett. 70, 1170 (1997).

${ }^{4}$ I. Larkin and Yu. N. Ovchinnikov, Zh. Eksp. Teor. Fiz. 68, 1915 (1975); [Sov. Phys. JETP 41, 960 (1976)].

${ }^{5}$ D. Y. Vodolazov and F. M. Peeters, Phys. Rev. B 76, 014521 (2007).

${ }^{6}$ A. Andronov, I. Gordion, V. Kurin, I. Nefedov, and I. Shereshevsky, Physica C 213, 193 (1993).

${ }^{7}$ A. G. Sivakov, A. M. Glukhov, A. N. Omelyanchouk, Y. Koval, P. Müller, and A. V. Ustinov, Phys. Rev. Lett. 91, 267001 (2003).

${ }^{8}$ P. Martinoli, O. Daldini, C. Leemann, and B. Van den Brandt, Phys. Rev. Lett. 36, 382 (1976).

${ }^{9}$ K. K. Likharev, Rev. Mod. Phys. 51, 101 (1979).

${ }^{10}$ E. Seynaeve, G. Rens, A. V. Volodin, K. Temst, C. Van Haesendonck, and Y. Bruynseraede, J. Appl. Phys. 89, 531 (2001).

${ }^{11}$ Micromagnetic simulation is performed by a public available code from NIST (http://math.nist.gov/oommf).

${ }^{12}$ A. Y. Aladyshkin, A. V. Silhanek, W. Gillijns, and V. V. Moshchalkov, Supercond. Sci. Technol. 22, 053001 (2009).

${ }^{13}$ J. Van de Vondel, A. V. Silhanek, V. Metlushko, P. Vavassori, B. Ilic, and V. V. Moshchalkov, Phys. Rev. B 79, 054527 (2009).

${ }^{14}$ A. V. Silhanek, J. Van de Vondel, A. Leo, G. W. Ataklti, W. Gillijns, and V. V. Moshchalkov, Supercond. Sci. Technol. 22, 034002 (2009).

${ }^{15}$ A. Yu. Aladyshkin, A. A. Fraerman, S. A. Gusev, A. Y. Klimov, Y. N. Nozdrin, G. L. Pakhomov, V. V. Rogov, and S. N. Vdovichev, J. Magn. Magn. Mater. 258, 406 (2003). 